\title{
Investigation into Current Industrial Practices relating to Product Lifecycle Management in a Multi-National Manufacturing Company
}

\author{
Ismael A. Essop[0000-0002-5583-0306] ${ }^{1}$, Richard D. Evans[0000-0001-6367-0560] ${ }^{1}$, Shan Wan[0000- \\ 0003-4368-9461] ${ }^{2}$,Muni P. Giddaluru[0000-0001-5894-1952] ${ }^{1}$, James X.Gao[0000-0001-5625-3654] ${ }^{1}$, \\ David Baudry[0000-0002-4386-4496] $]^{3}$, Sara Mahdikhah[0000-0001-5618-269X] and Mourad \\ Messaadia[0000-0002-3912-1788 $]^{3}$ \\ ${ }^{1}$ University of Greenwich, \{I.Essop, R.D.Evans, gm85, J.Gao\}@greenwich.ac.uk \\ ${ }^{2}$ Nanjing University of Science and Technology, shanwan23@hotmail.com \\ ${ }^{3}$ CESI/IRISE, Rouen, France, \{d.baudry,s.mahdikhah,m.messaadia\}@cesi.fr
}

\begin{abstract}
Product Lifecycle Management (PLM) systems have gained growing acceptance for managing all information relating to products throughout their full lifecycle, from idea conceptualisation through operations to servicing and disposal. This paper, through an in-depth exploratory study into a leading power generation manufacturing organisation, presents current PLM issues experienced by manufacturing companies, exploring three separate topics: 1) PLM, 2) Knowledge Management and Lessons Learnt and 3) Product Servicing and Maintenance. Following a review of published literature, results of the investigation are presented, analysing the responses of 17 employees interviewed. With respect to Product Development, it was found that information traceability is time consuming and change management requests take too long to complete. Results relating to knowledge management indicate that the Company operates a 'who you know' culture, but do aim to capture lessons learned on the manufacturing shop floor and assembly lines. Therefore, a prototype design is proposed to integrate the capturing of lessons learnt within the existing PLM system.
\end{abstract}

Keywords: plm, knowledge management, lessons learned, product maintenance and service.

\section{INTRODUCTION}

In today's modern business environment, Product Lifecycle Management may be seen to underpin corporate ability to meet customers' increasingly bespoke demands in a sustainable and competitive manner. PLM offers companies the capability of a framework to capture, store, retrieve, represent and re-utilise product and process knowledge in order to compete more effectively in today's knowledgeintensive Product Development (PD) environment [15]. PLM, however, is not solely a technological framework; it is seen to offer social and cultural dimensions which contribute to the strategy and competitive advantage of organisations.

PLM systems have gained acceptance for managing all information relating to products throughout their full lifecycle, from conceptualisation through operations to disposal. The PLM philosophy and systems, therefore, aim to provide support to an even broader range of engineering 
and business activities than merely PD. PLM was initially conceived as an academic concept to address the management of data, information and knowledge during product lifecycles, but subsequently has gained acceptance in industry to provide support to a wider gamut of business and engineering practices [36]. Traditionally, product information has been organised and generated by various business functions in an organisation. Functional areas, such as design engineering, manufacturing engineering, supply chain, sales and distribution, warranty and repair, and accounting all have information systems that reflect the needs of those functional areas [20]. The amount of data generated during PD processes is growing faster than ever before. For most aerospace, automotive and defence companies, to store data for 15-20 years is the norm, but creates a big challenge. In many instances, product data may be required to be stored for more than 50 years. To capture product information within an enterprise and between its partner enterprises, united, robust and flexible data models are required.

\subsection{Challenges facing Product Development}

The estimate of waste in design and engineering functions is often cited at $60-80 \%$ of the total design and engineering cost; this waste relates to manufacturing production, such as wasted motion, scrap (developing parts with design specification and drawings that are outdated), over production (designing parts that have already previously been designed), rework (designing parts that cannot be manufactured and must be redesigned), and material shortages (looking for drawings and engineering data and transportation waste - copying / moving drawings and engineering data) [20]. Translating a market opportunity into a new product requires approximately 15\% invention, whilst the remaining 85\% involves previously learnt processes that are often undocumented and undisciplined and, therefore, the need for improved knowledge sharing and the capturing of lessons learned during PD is currently at its highest.

\subsection{Research Approach}

The purpose of this paper is to report on an Industrial Investigation which was conducted in a leading power generation manufacturing company in the UK between October 2013 and March 2014. For the purpose of this report, the organisation is referred to as 'The Company'. The aim of the investigation was to gain an understanding of the Company's current practices and challenges in relation to: 1) Communication between internal colleagues and external stakeholders; 2) The Product Development Process; 3) ICT Tools currently used within The Company to assist with PLM; 4) Knowledge Management (KM) and Sharing; and 5) Product Maintenance Management.

The methodology used during the investigation was informal, audio-recorded face to face interviews lasting between 60 and 90 minutes. In total, 17 employees from 5 different departments were selected for interview. Participants had a wide range of experience in design, manufacture and maintenance and included the Plant Manager, Maintenance Engineers and Assembly Line Operatives. Interviews were conducted on an individual basis by a panel consisting of two PhD students and one post-doctoral research fellow. The interviewers followed a standardised questionnaire, which asked participants a variety of open-ended and closed questions to identify the current methods, practices and tools employed within The Company for KM, PLM and product maintenance management; the findings relating to these questions are summarised in this paper.

\section{REVIEW OF PUBLISHED LITERATURE}

\section{$2.1 \quad$ PLM}

Product lifecycle management is a set of capabilities that enable an enterprise to effectively and efficiently innovate and manage its products and related services throughout the entire business lifecycle, from conception through recycling and disposal.

Most engineering information systems store and retrieve data in different forms and using different file types. Specifically, in the computer-aided engineering and design field, the number of files associated with individual products, parts, components and their associated Computer-Aided Design/Computer Aided Engineering/Product Data Management and Product Lifecycle Management 
(CAD/CAE/PDM/PLM) systems and system users, has reached a level of vast complexity and little transparency; this is due to the fact that current file systems are still based on traditional hierarchical directory structures. In such file systems, users are required to organise their data in hierarchical structures. In such structures, specific path and file names have to be determined and correctly entered in order to store and retrieve data. Unfortunately, these traditional hierarchies often do not scale to large data collections and to the fine-grained classifications required. As the data classification hierarchy grows, files are increasingly likely to be assigned several different classifications that are all linked to only one associated file location. To locate and retrieve a particular file at a later date, system users must remember the one specific classification that was originally chosen.

The PLM concept links different stages of PD, including CAE, CAD, PDM, Manufacturing Process Management (MPM), Enterprise Resources Planning (ERP) inter alia in a unique numerical chain [17]. The target is to better answer questions relating to time-to-market, cost, and quality criterions. In fact, there is no unique tool or method which allows the management of a project for the development of a product; it is a very difficult task because of the vast amount of information which comes from different sources in the process of product development. PLM is collaborative in nature and aims to integrate all partners and associated knowledge efficiently. When implementing PLM, organisations need to consider the collaborative process and how it can be optimised by allowing up stream integration of data, resources and knowledge. PLM systems are IT application frameworks that are widely acclaimed for supporting this objective, along with enhancing KM capabilities and coordination among the functional areas involved in New Product Development (NPD) [7].

In general, PLM systems integrate [46]:

- $\quad$ Systems and technologies to support design activities (i.e. visualization/viewing applications, CA-X integration, PDM, engineering change management tools, configuration management tools etc.), in the context of interdisciplinary and distributed teams (i.e., data exchange and collaboration technologies, design coordination tools);

- $\quad$ KM systems (i.e. document management, content management systems etc.);

- $\quad$ Project management and workflow management tools; and

- Systems and technologies to support relations through the supply chain (i.e. customer-oriented and supplier-oriented applications, or information tracking systems).

Recently, business leaders have recognised the need for wider collaboration and integration for sharing of product information within business that had previously been spread globally and across supply chains. They have further realised that they are far from reaching their goal. One reason for this is that until recently, information technology tools and strategies were not up to the challenge of effectively sharing information across such a complex network in a flexible and robust manner. This is about to change due to the convergence of three important technological developments: (1) maturity of standardized product data and product meta-data models, and standardized engineering and business processes; (2) emergence of Service-Oriented Architecture (SOA) for information sharing; and (3) availability of robust middleware to implement them [46]. A PLM infrastructure that combines the benefits of these three developments, which have converged almost in perfect harmony, is bringing us closer to realising the dream of business and technical integration and partner collaboration.

\subsection{Knowledge Management}

Since the 1990s and given the ever more challenging economic conditions experienced by companies today, there has been increasing recognition that one of the most valuable resources owned by an organisation is its employee and organisational knowledge. Prior to the 1990s, Porter and Millar [37] suggested that the key to a company being successful was in the information it possessed. Nowadays, it is believed that employee knowledge is the key to corporate survival and growth; companies need to maintain and make better and more informed use of their employee, partner and organisational knowledge [16]. In 1991, Nonaka [32] predicted that successful companies will be those that create, capture and make best use of their knowledge and then apply it to new innovative PD. The need to manage and develop this knowledge effectively has increasingly been acknowledged in order that organisations may respond to the challenges presented in today's dynamic and complex business environment and to overcome economic uncertainties [39]. 
Research [32] shows that there is a distinction between employee knowledge and organisational knowledge. Tsoukas and Vladimirou [48] explained that employee knowledge is the "individual capability of a person to draw distinctions, within a domain of action, based on appreciation of context or theory, or both" whereas organisational knowledge is the "capability of members within an organisation to draw distinctions in the process of carrying out their work, in particular, concrete contexts, by enacting sets of generalisations whose application depends on historically evolved collective understandings"; for the purpose of this study, research is carried out at both employee and organisational levels.

Nonaka [33], described organisational knowledge as the information flow among the various resources within a company; it is the knowledge that is captured through IT systems, organisational processes, products, company rules and corporate culture. Knowledge within an organisation can include workers' experiences and skills, which may be stored in the minds of the employees (tacit) or could be recorded in physical documentation (explicit), which is available for all employees to view and re-use. Myers [31] adds that organisational knowledge is "processed information that is embedded into routines and processes that enable action". Research [32] shows that organisational knowledge and the management of that knowledge are critical to the success and competitive advantage of an organisation [34]. Pillai and Min [35] state that knowledge plays a critical role in the successful management of an organisation's supply chain. Ross [42] states that although supply chain members are independent entities, they still need to form inter-firm and intra-firm alliances, which make them mutually dependable on each other to meet common goals. All parties in a supply chain need to work together collaboratively to solve problems and develop innovative products to meet the demands of customers. Consequently, knowledge sharing should become an integral activity within any supply chain. In 1999, however, Liebowitz [24] explained that knowledge cannot necessarily be managed and instead is a set of activities that companies must practice in order to achieve competitive advantage; these activities were described as: Knowledge Creation; Knowledge Valuation and Metrics; Knowledge Mapping and Indexing; Knowledge Transport; Storage and Distribution; and Knowledge Sharing [6].

The management of employee and organisational knowledge is becoming increasingly important for the survival of manufacturing organisations [2]. Researchers [5] believe that, by sharing formal organisational knowledge with partners in the supply chain, companies are now able to increase performance and ultimately be successful in global marketplaces. Empirical research [32] suggests that organisations that actively share and make use of collective knowledge with partners in the supply chain become more productive and are more likely to survive than those that withhold knowledge within their company. The external knowledge of a company includes customer, supplier and competitor expertise. Organisations which are able to tap into that knowledge base increase significantly their chances of succeeding in competitive markets. However, there are numerous barriers to creating a culture for sharing between these entities and it is recognised [1],[41], in particular, that the activities of knowledge capture and sharing face numerous barriers typically relating to either social factors or the technology adopted or a combination of both [6]. Barriers include:

- Businesses often cannot identify what is known within their organisations and, consequently, best practices, expertise and knowledge and skills cannot easily be applied and transferred;

- The physical layout of an office space or the hierarchical structure of a company may be counterproductive to knowledge capturing initiatives [41];

- Individuals often perceive knowledge as power that can allow them to advance within an organisation and, therefore, are not prepared to share [1];

- Individuals often do not have the time to contribute knowledge to others, if they are working, for example, on busy production or assembly lines [41];

- Employees within organisations often will not share their tacit knowledge for fear that other individuals will take credit for the knowledge they have previously shared [41]; Choi et al. [10] suggested that trust and reward mechanisms are possibly more important than technical support in isolation for encouraging knowledge sharing;

- Organisations often do not share knowledge with external partners for fear of divulgence of confidential information, including corporate technologies, pricing schedules, customer databases and processes [31];

- Language and cultural barriers, including the differences in cultures between North America, Western Europe, Japan and Spain. Desouza and Evaristo [14] argue that Spanish and Japanese 
employees are far more open to the sharing of knowledge, whereas employees in North America and Western Europe were more reserved when it came to sharing their tacit knowledge and wanted confirmation that the knowledge would be controlled and not be misused;

- A lack of consistent policies across businesses often hinders the successful management of knowledge management systems [22];

- The interoperability of new knowledge management systems into existing IT infrastructures and compatibility issues with current or legacy systems;

- Organisations often do not offer sufficient training or explain the benefits of new knowledge management systems; this causes difficulties due to many employees being unfamiliar with the new system installed; and

- Employees leaving the business; Preiss [38] recognised that important tacit knowledge will be lost if an organisation fails to capture it before an employee leaves. Similarly, if an employee stores work locally on a personal hard drive rather than in shared folders or cloud-supported networks, where others have been granted access, then explicit knowledge already stored may not be captured and made available to others, including new employees.

Finally, it has been acknowledged by Myers [31] that organisations which share similar philosophies and corporate culture have a far greater inclination to share knowledge with each other. They further added that organisations which have made investments in supply chain partners (e.g. financial, equipment, facilities) are more open to knowledge sharing because they have an interest in the other party developing their knowledge.

\subsection{Lessons Learned}

This sub-section will review relevant research relating to Lessons Learned (LL). A statement that is increasingly cited, both in academia and industry, as a comprehensive definition of LL is one used by the American, European, and Japanese space agencies: "A lesson learned is a knowledge or understanding gained by experience. The experience may be positive, as in a successful test or mission, or negative, as in a mishap or failure. Successes are also considered sources of lessons learned. A lesson must be significant in that it has a real or assumed impact on operations; valid in that is factually and technically correct; and applicable in that it identifies a specific design, process, or decision that reduces or eliminates the potential for failures and mishaps, or reinforces a positive result" [44].

Many prominent Engineering organisations nowadays collect lessons learned. The early pioneers of this initiative have been space agencies and military organisations. A significant part of the work related to simulated exercises, after which members are asked to describe what they learned and store this in generic or dedicated information repositories. A number of researchers [27] regard LL practices as building blocks of learning in organisations and is an agent of knowledge creation. LL allow the capture of individual and group experiences into organisational knowledge. Therefore, organisations need to have what Cooke-Davies [12] describes as an effective means of learning from experience on projects which combines explicit knowledge with tacit knowledge in a way that encourages people to learn and to embed that learning into continuous improvement of project management processes and practices.

When lessons learned occur at the end of projects, they are known as Post-Project Reviews which is one of the most common approaches for the capture of project knowledge [11],[43]. This exercise provides opportunities for cross-functional teams to share, discuss and express their experiences in especially organized meetings.

Manufacturing companies are often pressured to work 'faster, better, cheaper' than their competitors, and they are increasingly looking at streamlining their NPD processes. During the early design phase, engineers need to access specific knowledge that is often captured in LL from previous projects. In global companies, where both knowledge and expertise are geographically distributed, it becomes a major challenge to locate experts and tap into their know-how'.

From case studies described in the literature review, there were a range of ways that lesson learned could be captured, from fairly unstructured manner to detailed methods, that help with information retrieval after the project have long been completed [11],[19],[21]. 
Lessons learned are described and captured in a variety of forms. A structured LL activity should comprise of a set of contents, such as the learned strategy, how it was learned, and how it can be applied for reuse. There has been a number of research studies conducted which explored ways to describe and capture LL in a structured format. In order to support reuse, lessons must be presented in a timely and contextual way.

Richter [40] proposes a Case-Based Reasoning system that tracks each task included in an engineering project and searches its database for lessons that are applicable to each task. Milton [27] suggested a LL structure, including: context, description of the event, root cause of the problems, lessons identified and suggested action while Chirumalla [9] proposed a seven-step representation of lessons learned, consisting of: (1) lesson learned statement, (2) working context, (3) task description, (4) what went wrong or what went well, (5) lessons learned, (6) lessons learned measures, and (7) applicability and delimitations.

\subsection{Product Service System and e-Maintenance}

According to [49], maintenance costs account for $15 \%$ to $60 \%$ of the total production cost of a product, with the maximum being experienced for complex products. In the Power Generation Industry, a product's life can span from 30-50 years; this, therefore, means that the maintenance, repair and servicing of them is extremely important either on service strategy or KM from a PLM point of view.

Product Service Systems (PSS) were first introduced in 1999 [18] in order to manage product and service information together, in order to create value for both customers and manufacturers. Goedkoop et al. [18] defined PSS as "a system of products, services, network partners and supporting infrastructure that is economically feasible, competitive and satisfies customer needs. It offers dematerialised solutions that minimise the environmental impact of consumption"; this definition means that PSS not only contains product and service information, but also includes stakeholders throughout the product lifecycle and supporting technologies, information/knowledge which makes the product suppliers more competitive, the customers more satisfied and society more sustainable. There are three commonly accepted classification for PSS [18]:

- $\quad$ Product-oriented PSS, whereby physical products are sold with added after-sale services, such as maintenance, repair, monitoring and management;

- Use-oriented PSS, whereby product vendors/manufacturers have ownership of the physical products, while the usage of the product is sold to the customer; the owners are responsible for the product's condition, such as its maintenance and timely installation of upgrades; and

- Result-oriented PSS, whereby customers purchase functional results or capability of a product provided by a vendor under an agreement. A good example for this is that customers purchase laundered clothes as a result of buying a washing machine .

According to the characteristics of maintenance and service in power generation, they belong to the product-oriented PSS. However, little research has focused specifically on PSS in the Power Generation Industry (PGI), although there are researchers exploring PSS in other industrial sectors, such as automotive [25] and manufacturing [50]. Wan et al. [51] provided a method of developing an eMaintenance systems based on knowledge management to improve maintenance efficiency of manufacturing systems in a Power Generation company. Research relating to maintenance and servicing in power generation has historically focused on maintenance scheduling. For example Dahal and Chakpitak [13], adopted a meta-heuristic approach to optimise maintenance scheduling problems, while Badri et al. [3],[13] scheduled generation maintenance work, considering system indices, such as security and reliability; other research [47],[23] has optimised the maintenance strategy for power generation systems.

Due to power generation equipment being located in remote and isolated places, product service engineers often obtain as much information as possible before travelling to another place to fix the problem the ability of service engineers to access all relevant information and knowledge is very important. E-maintenance is a strategy that can reduce the influence from geographical barriers and improve the management of maintenance knowledge and its process [8]. Through the adoption of an e-maintenance platform, maintenance actors could communicate and work together through the maintenance process and information they acquire could be retrieved far easier to support decision 
making [8]. Chebel-Morello [8] provided a framework on how to reason the previous knowledge by using ontology to model equipment and failure, and then using the Case based Reasoning (CBR) method to get the recommended solution.

Muller [30] provided a review on the current situation and challenges of e-maintenance; although this was written in 2008, it still offers a guide to the future development of e-maintenance platforms. According to [30], the ability of organisations to achieve knowledge-based maintenance operations is still one of the most urgent industrial requirements.

\section{INDUSTRIAL INVESTIGATION}

This investigation was conducted within a global manufacturing company based in the UK. The organisation has 4 main business units: Engine, Power Generation, Components and Distribution. The business consists of 17 company-owned distributors and 10 joint ventures, covering 90 countries and territories through 233 locations.

The investigation was conducted within the Power Generation business unit of The Company, exploring 4 main research areas: 1) Product Information management, 2) Knowledge Management, 3) Collaboration and 4) Maintenance Management. The methodology used during this investigation is detailed in Section 1.2.

\subsection{PLM}

The Company uses 3D and 2D design tools for product development and Product Data Management (PDM) for storing CAD data. The PLM system is used for storing the released engineering product information. There are work flow processes designed to carry information from CAD to PLM system and a life cycle process is used for design approvals. As part of the investigation the following questions relating to product information were asked to the interviewees, with the aim of understanding how product information is stored and how it flows between different systems.

- What PLM systems do you use?

- Could you explain how your department manages product information and knowledge?

- Have you encountered any problems with the management of information?

- Does all other business units use same systems? If not how do you manage data?

- How many systems do you use for storing product information?

- What type of new product development methodology do you use?

- What are the big challenges that you are facing in data integration in upstream and downstream systems?

- What is the longest life of your data approximately?

- How your data is shared between other business units? What tools you use for this purpose?

- Does your data is contained in different languages since its global company?

Interviewees concluded the following: Product information is stored in different systems by different departments in the process of NPD and continuous improvement processes. The company uses 3D and 2D CAD design tools to create and maintain product engineering information. All native CAD data is stored in a PDM system called pdmlink. The drawings from pdmlink are released from/to the PLM system. This process converts drawings to PDF format and stores them in the PLM system; there is limited integration between the PDM system and PLM system to transfer the PDF file and Engineering Bill Of Material (EBOM) to the PLM system.

The PLM system has got an embedded life cycle approval process called 'Engineering Change Order' that flows to Responsible Design Engineer, Product Line Manager, Chief Engineer, Standards team, engineering data management group for approval. PLM then integrates with downstream Enterprise Resource planning consisting of product configuration management, manufacturing, supply chain management, order management, customer services, after market and more. A large amount of data is generated during the process of NPD. Apart from design data, a lot more product information is generated during product theoretical validation (Final element analysis, Aero dynamic analysis, simulation details etc.), configuration design (General BOM, product specifications, module specifications, configuration bill of material, parts classifications etc), manufacturing (manufacturing 
planning data, tolls and equipment information, work instructions, manufacturing BOM, routing information, NC programs, flow charts, maintenance information, shop floor instructions, process plans, tools and fixtures etc.), supply chain management (supplier information, parts cost, supply strategy data, purchasing information etc.), testing (test planning information, testing tools and its maintenance information, test reports etc.), order management (customer order details, customer information, pricing data etc.), inventory management (raw material and finished goods data etc.), logistics (goods in / out, shipping information etc.), finance (product pricing, sales, invoices, purchase orders, profit/loss data etc.) and miscellaneous (patent, regulations, service manuals, spare parts information, technical publications etc.).

The above information is stored in different systems. Engineering standard work, test results, Analysis Led simulation analysis is stored in local servers, while CAD data is stored in pdmlink. Product release data, EBOMs and change management is stored in PLM. Manufacturing data is stored in a few different systems, including CAD data management, ERP and shared server spaces. Product testing result data is stored in access-controlled shared servers. Order management, sales, customer and supplier information is stored in an ERP system. Logistics data is stored in a data warehouse management system. It can be seen that the lifecycle of a product is stored in many systems; this is only one business unit and one locations data. If we imagine how much complexity will be there in connecting cross functional and cross sites globally. The company has a presence in more than 10 countries worldwide, in design and manufacturing; each site produces different ranges of power generation products, so the data is very big and tracking this data in different systems makes more completed across globally, as can be seen in Figure 1.

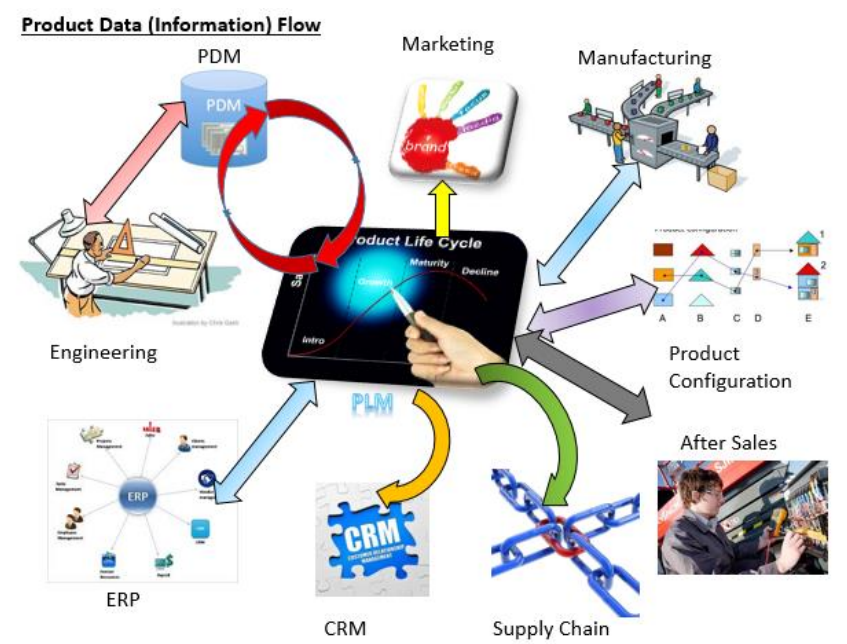

Fig. 1: Product Information Flow in the Industrial Case Study.

In this section, we provide an analysis to the responses gathered about how PLM is managed in the organisation. The company uses multiple systems to store product information in the process of product development. In this study, data flow analysis was limited to 3 systems: CAD, PDM and PLM. The following conclude interviewee responses:

- Product information traceability: It is difficult or sometimes not possible to trace product information. One example is that CAD data is stored in a PDM system and the same released data is stored in a PLM system, since they are two different systems, the revisions of the same files may not be same. Revision control is manually maintained and it is not foolproof. Another example is regarding test results, theoretical analysis, where engineering standard work data is stored in shared drives and relating this information to a particular product takes lot of time and is sometimes very difficult; 
- Integration issues: Data from CAD system goes to PDM and from PDM it is stored in PLM. Due to compatibility issues between the two systems, data will fail to transfer from one system to another. One example is transferring EBOM from PDM to PLM;

- Cross functional Data Sharing: Since the company uses different systems for the same functions in different business units or different locations. This makes it complicated to share product information within the same company due to compatible issues. One business unit uses E-Matrix PLM system to store both CAD and PLM data, while another unit uses PDMLink for CAD data management and E-Matrix for PLM functionality;

- Common standards issues: As a global company with different locations, each business site uses their local standards as per requirement. For example, some sites use metric while some uses inches as units. When the organisation integrates these products to sell in other parts of world, they need to consider the customer local requirement and make necessary changes to release the product;

- Change management: Engineering change management takes a lot of time in the approval process. Since the life cycle goes through many individuals for approval, it takes a lot of time to be released; this also depends on system performance;

- Supplier integration: Since the company's suppliers cannot access the internal PLM system, data sharing is a manual process; this leads to suppliers working on incorrect versions or part drawings, which have subsequent revisions. The current PLM system adopted by the company does not have sufficient functionality to share data with supplier securely with traceability;

- Legacy Data: While implementing the PDM and PLM systems, all legacy data is stored as is. With new systems being introduced, this data requires many changes to make it compatible with each system; this takes a lot of engineering workload, which leads to an increase in the product development cycle; and

- $\quad$ Reuse of existing data: The product data is not stored in a systematic or logical manner, which makes it very difficult to reuse for new or similar product development projects.

Product information is generated and maintained in many different systems in a typical manufacturing company and this company is no different. The main issue we found in this investigation is that information traceability is time consuming and change management life cycle takes too long to complete. Maintaining and applying common standards globally are challenging. There are numerous numerous hurdles to overcome to try to address the legacy data which is required prior to a PLM implementation. Furthermore, there is no complete solution from PLM implementation in how to deal with the existing legacy data.

\subsection{Knowledge Management}

This section answers the following questions relating to knowledge management, which were asked of interviewees during interview:

1) How do employees within the Company currently identify best practice knowledge for specific manufacturing problems?

2) How does the organisation currently store best practice knowledge?

3) How Do You Currently Share Best Practice Knowledge with dispersed and co-located colleagues?

4) What knowledge management tools are currently used within the Company? How frequently are these tools used?

Firstly, in relation to question 1, employees explained that for every manufacturing project, a set of work instructions are produced detailing relevant knowledge in relation to the design, manufacture and assembly process for a complete product. This explicit knowledge is stored on an internal database, which is the first point of reference for operators working on the Assembly line. The database is accessible directly from the shop floor using touch screen monitors and product designers and change engineers can modify product documentation remotely. It was reported that the company promotes a "who you know" culture, whereby employees are encouraged to ask colleagues to share knowledge and, if that colleague does not have the required knowledge, ask if they can suggest someone who does. If it is not possible to find required knowledge internally, employees are 
encouraged to use external resources, such as commercial search engines. There is no system in place for the storage, identification and retrieval of explicit knowledge of employees. Currently, information is captured in word document format when users are completing work processes, although no further action is taken to convert it into explicit knowledge. Finally, if someone experiences a problem, they are encouraged to visit Team Room, an intranet accessible by colleagues working within the same project group, and ask questions via an instant messaging tool.

In relation to Question 2, employees confirmed that best practice knowledge is typically stored in spreadsheets and then made available via the EASE touch-screen system. Knowledge is occasionally input into 'Team Room', although this is not considered a standard practice. It is recommended by the corporate IT department that employees store all manufacturing documentation on a shared server or on the internal intranet, but this does not always happen and often information is stored locally on personal hard drives. During the NPD process, the company aims to capture lessons learnt during projects at the product closure stage. This activity informs colleagues of issues which arose during manufacture, testing and execution and is stored for future reference. After product delivery, however, there is no requirement to update this information (e.g. when a product is repaired in the field) and inservice experiences are not added to the lessons learnt document. It was stated that the company is currently seeking to adopt paperless working and a new system called EASE has recently been introduced, which offers a Touch-screen workstation for assembly line operatives. The system stores all product development project documentation, from work instructions to training manuals and all critical product characteristics. The company also operates a system called "QSI", where all training materials are stored; this is an electronic system controlled through revision changes and approval processes and allows for the preparation of operator instructions when new products are introduced.

In relation to Question 3, it was confirmed that the Company does not currently have a standardised method for sharing knowledge relating to best practices, although employees commented that they try to make their knowledge as easy as possible to understand; to this end, they aim to remove from documentation any unnecessary technical jargon for stakeholders who do not have a technical background. It was revealed by interviewees that they usually use e-mail as their preferred communication tool when sharing knowledge. An internal intranet facility, called Team Rooms, exists to support project work and it is the responsibility of functional team leaders to ensure effective communication between team members.

Finally, in relation to Question 4, which aimed to identify specific knowledge management tools employed within the organisation, none were identified. For the recording of maintenance issues, a Tcard system is employed where users note down in paper format any problems or issues being experienced with manufacturing and assembly equipment. Interviewees reported that the company relies heavily on e-mail and corporate management are trying to encourage employees to collaborate more pro-actively and, thereby, share greater knowledge through informal communication. It was confirmed that the Company provides an instant messaging system for informal communication and a corporate intranet is available to transmit company-wide information. It was pointed out that project groups are also able to create and manage their own intranet sites for communication purposes, although no micro-blogging functionality is available to facilitate business processes.

\subsection{Capturing Lessons Learned during PLM}

The interviews yielded a large amount of information relating to the methods used by the company for capturing Lessons Learned. This section evaluates the results obtained by the investigation, focusing specifically on LL activities during NPD.

"But lessons learned for me, particularly in NPD, we talk about KM, it has got to be up there. That's the only way you are going to gain knowledge. And continually to improve on the launch, to the next launch, to the next launch, is learning lessons from the previous ones. Once the project is completed, we do have a lessons learned (activity), but what we are not doing, not only us, but most of the industry too, we are not tracing back when there is an issue with the original document. Once we close the project, we tie up all the things, from an Engineering point of view, we have done all the work, we have released the product, my perception is from a business perspective, it is a tick in the box activity." Six Sigma Quality Champion. 
The above statement highlights the fact that LL activities need to be incentivised so as to promote its use and reuse within the organisation. Currently, there is no framework in place to identify best practices. LL are recorded, but then are often lost in the system. Clearly, there is a need to analyse these experiences in an effort to create a feedback-loop in the development process. Since no feedback loop has been established, many employees are unaware who or which department/unit may benefit from the insights and experiences captured.

"It's part and parcel of the sign off. So within the 180 days, so the lessons learnt page, slides, depends on how many lessons learnt you've captured, depends on how long they actually are, everyone is different. Some people are quite prescriptive with what they've learned from one another learn, others will just have bullet points." Staff Manufacturing Engineer A

This statement confirms that LL are captured at the end of each project, but that they have a fairly low priority. Once the company's employees have already been assigned to new projects it is challenging to remember the important issues encountered on previous projects, as there is a delay between the occurrence of the issues and the capture of the experiences.

Interviewer: Yes. And is there an example template which you can use to fill in? Respondent: No, at the end of the template, it says: lessons learnt, it's blank.

Interviewer: Interesting, and who is able to record the lessons learnt, can everybody who worked on the project contribute to the lessons learnt, or it's just the...?

Respondent: It's just the belt. The guy who is leading the project obviously there would be five or six team members which come from a diverse background, so you get different inputs, not just looking at one part, the manufacturing part, or purchasing part, you've got other people coming in and give their feedback and their comments, but generally it's the belt." Staff Manufacturing Engineer A.

The above is a typical example where LL have been put into place, but are almost never systematically applied, as confirmed by Milton [6]. It has become apparent that the LL process lacks rigour. The quality of reports vary between projects and are often influenced by factors, such as budget and the belt's interest.

"We have six sigma to record the lessons learnt from the different projects and put it in the document in a file, so for the new projects, it would be useful to see what happened in the past and get off some of them." Manufacturing System Engineer B.

It is evident that while there is a process for explicit knowledge to be captured, the current system used does not allow for the capture of tacit knowledge. The communication is one-way and is very much individualised and, therefore, it is not conducive for sharing and distribution. There is currently no organisational policy for how this knowledge should be shared and fed back to other departments.

During the investigation, it was observed that a number of interviewees were unaware whom within the organisation or department was accountable for ensuring that lessons are identified and recorded properly. The identification of lessons learned varied from project to project. Some staff held free-format meetings of the project team to discuss what has been learned during a project, while others designated an individual to write an end of project review document.

The current PLM system used within the company does not have the level of customisation to capture LL during NPD. Currently, LLs are only captured at the end of the project, and is done in a very unstructured way. Consequently, this information is unlikely to be reused during NPD. There is a need to analyse these experiences in an effort to create a feedback loop to the development process. Since no feedback loop have been established in the company, many engineers are unaware who may benefit from the insights and experiences captured. Many prominent Engineering organisations nowadays collect lessons learned. A number of researchers [21],[40] regards LL practices as building blocks of learning in organization and agent of knowledge creation. They allow the capture of individual and group experiences into organizational knowledge. Therefore, organizations need to have what CookeDavies [12] describes as an effective means of learning from experience on projects, that combines explicit knowledge with tacit knowledge in a way that encourages people to learn and to embed that learning into continuous improvement of project management processes and practices. 
Based on the requirements highlighted above, we are proposing the design of a Lessons Learned system that can be later added to the company's PLM system. The design takes into consideration the generic aspects of framework proposed by other researchers such as Chirumalla [9] and Milton [28]. They are presented as wireframes shown in Figures 2 and 3.

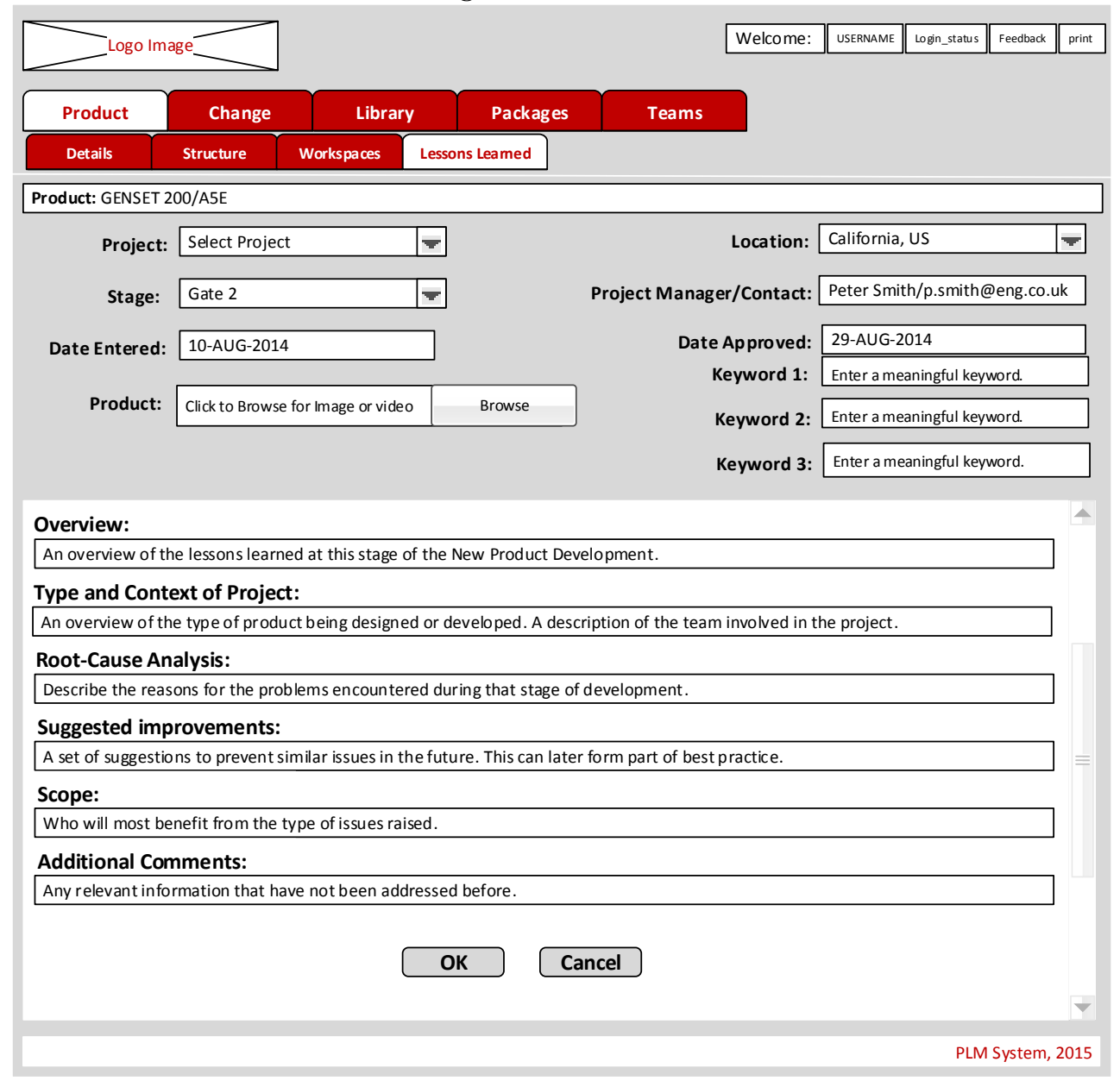

Fig. 2: Data Entry Form for Lessons Learned module.

The schematic aims to provide an interface that will allow geographically dispersed engineers to search and retrieve lessons learned on past projects. This operation is extremely valuable as this will help avoid repetition of past mistakes, and provide an opportunity to share best practices across the different business units within the company. In a large multinational company, it is often challenging to search for the relevant person that was engaged with a particular project, therefore we have provided a contact information field. The keyword fields will significantly improve information retrieval.

Figure 3 shows the second wireframe which represents a search result of a particular product at a certain stage in the product development process. The assigned staff can retrieve an image or a video of the part, and a detailed narrative of the lessons learned process is presented to the user. 


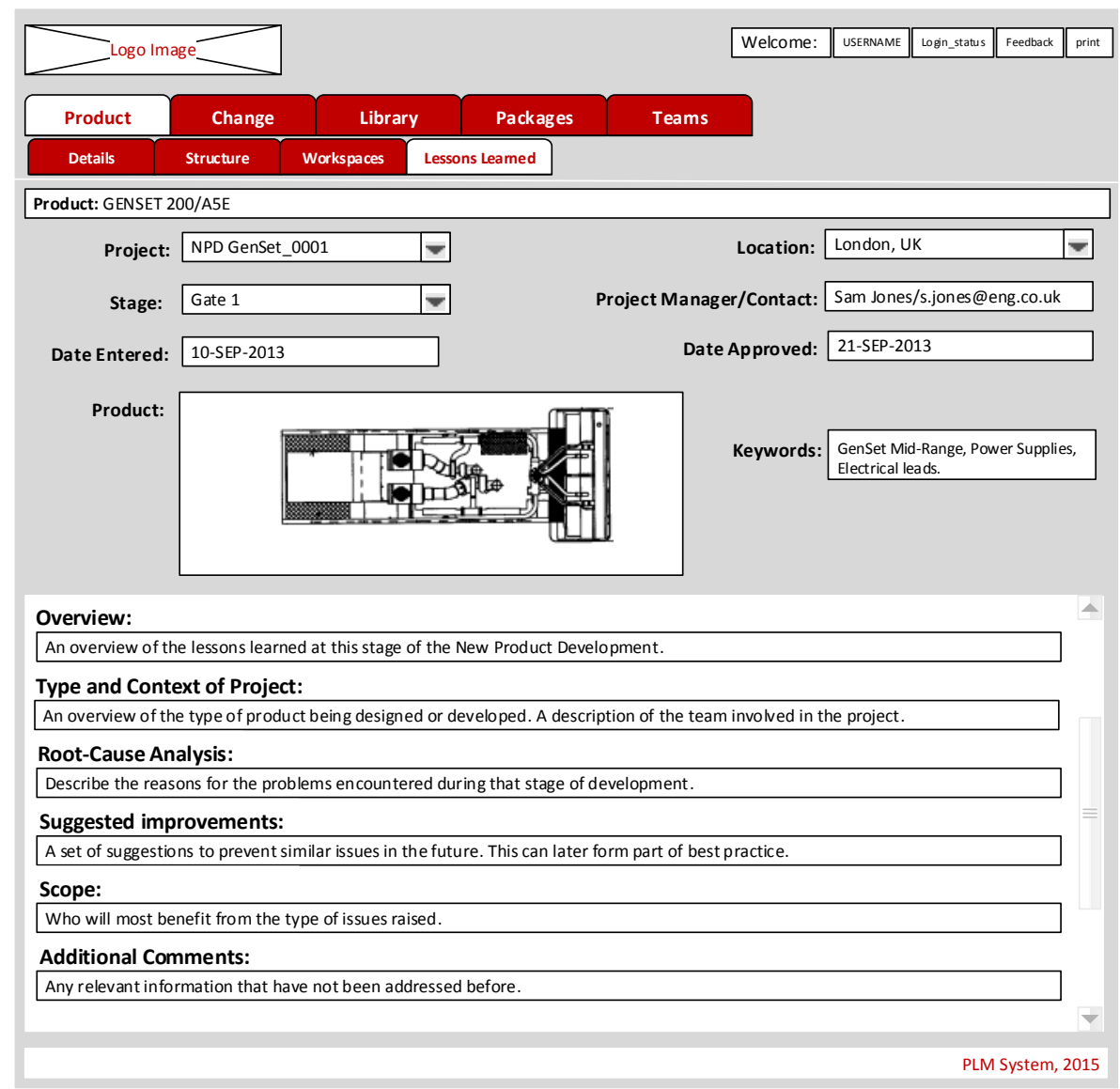

Fig. 3: Search Form with Results in Lessons Learned Module.

\subsection{Product Service Management}

This section provides the answers to the questions of product service process raised during the investigation. This is then followed by an analysis of the responses gathered. The questions asked during interviews relating to product service management included:

- Does your company have outsourced service partners? (To get an idea of whether they encounter the knowledge sharing problem with their partners)

- $\quad$ Where are the service engineers based? (To know their work mode)

- How long is the service provided to the customers?

- How do you get service requests from customers and give them feedback?

- Does the product service department give feedback to the product design department? (To get an idea of how they communicate from the PLM point of view)

- How do you currently manage problems experienced by customers? If there is a system that manages these problems, root causes and their historical solutions, how are they retrieved and reused?

- Can you obtain a recommended solution from the system once you type several retrieving words? If yes, following the solution, does it provide procedures and steps to solve the problems? If not, do you think it is necessary to get the recommended solution to guide engineers work?

According to the questions asked during the investigation, there were several key points obtained:

- The Company does have a product service department; product service engineers will go out with diagnostic tools and plug it into their control panels and try to understand what's gone wrong 
with a product. Once a diagnosis has been made and the engineer is able to locate the fault and what caused it, they would then either fix the part or replace it;

- $\quad$ Service engineers are globally dispersed; they are mobile workers who travel a lot to other places in the world, whenever a call is received. They will go and get the product back up and running, either as a short fix, and then they work through a long term implementation strategy;

- When asked whether the service is provided lifelong, interviewees stated that the product is sold with a warranty period, which can be extended by the customer. The Company offers 5-6 different warranty levels for customers to choose from: a standard package and others with differing options, ranging from 1-6 years. If something goes wrong outside of the warranty period, engineers still go to repair it, but the customers would incur a charge;

- A common platform exists between the Company and its customers, which is named Customer Service Management System (CSMS). The system allows customers to input their problems and the support engineers to share information with product engineers in collaboration. A database within the Company is called Issue Tracking System (ITS), which receives customer issues. Once input into the ITS, issues are followed up by service engineers. Once the customer request is received, the service manager will raise the respective documentation and then distribute the tasks to different engineers whom are located close to the customer experiencing the problem; the ITS database records all problems which can be retrieved once receiving a new customer request, by going through the previous activities, service engineers then go out to the sites and fix the problems and report back to the system once the work has been completed to close the process;

- Apart from the ITS, there is another system called Before In Service (BIS), which is used before a product is commissioned and if there are any problems. For example, if the product has been rattling during transit, a BIS is raised for the service engineers to distinguish between product fatigue failures and assembly issues;

- $\quad$ Feedback from the product service department is shared with the product design department. Product support engineers physically come to talk to the product design engineers. There are two parts of engineering in the Company: product development and product support. The latter are the eyes and ears to the ground, which carry out short and long term fixes, but they always come back to product development to seek collaboration, because it is the product development department that own and control product design; and

- The CSM system is not intelligent, employees can retrieve lessons learnt information stored in the system. Once retrieved, similar issues and their solutions are displayed for the engineers to reuse. However, the system does not select the best solution; this creates a manual process which relies heavily on the knowledge and experience of the engineer. In the power generation field, it doesn't matter how detailed the description of the solution is, it is the qualification that matters because the person cannot do the job if he hasn't been qualified to do the job; this means that the organisation is extremely reliant on the knowledge and experience of the engineer and the qualifications of the person doing that job.

According to the responses to the questions, it can be established that the Company employs a Customer Service Management System (CSMS), which is used during service processing. Due to the requirement from the product support department, for product design information, a product service system seems necessary to be implemented in The Company. According to the definition of PSS, given by Goedkoop et al. [18], a PSS is "a system of products, services, network partners and supporting infrastructure that is economically feasible, competitive and satisfies customer needs. It offers dematerialised solutions that minimise the environmental impact of consumption"; this definition means that a PSS should not only contain product and service information, but also include stakeholders throughout the product lifecycle and the supporting technologies and information/knowledge which makes the product suppliers more competitive, the customers more satisfied and society more sustainable. The current CSMS system stores lessons learned, but does not store it using a formalized method, which leads to difficulties in knowledge retrieval efficiency and effects, whenever knowledge is needed [29].

Based on the comparison between the industrial investigation and the literature review of PSS and e-maintenance, it can be seen that there is a requirement for the PGI to apply knowledge-based maintenance management systems to enhance the optimisation of maintenance operations and the efficient reuse of employee knowledge. 


\section{SUMMATIVE ANALYSIS}

The Company has implemented a PLM system for the first time 6 years ago. From the investigation, we found that there are some basic issues that need addressing in order to maintain the product information. During the implementation the team mainly concentrated on CAD data management, and its output (drawings) and only one level of bill of material. There was no plan in how to deal with the legacy data, which lead to have issues in new system. It was a missed opportunity to relate other product information generated in and outside the engineering function into the PLM system. Whilst the design of change management system is very robust, it is taking a lot of time to complete the cycle. There is scope to address this by doing some customisation.

The industrial investigation confirmed that the Company does not currently have any formalised method for sharing explicit employee knowledge. Some tools and procedures exist to facilitate knowledge exchange, but the organisation lacks a standardised process for the capture, management and sharing of explicit employee and organisational knowledge. Given the relatively flat organisational structure and a corporate culture of "who you know", employees are expected to seek out knowledge from identifiable colleagues. This is frequently completed on an oral face-to-face basis while written documentation is shared. However, it may be concluded that the Company is failing to embrace current technologies to facilitate and enhance knowledge sharing.

From the investigation, there is a general awareness of the inherent benefits of lessons learned in NPD and some momentum would be welcome to promote its use across cross-functional teams. There is a need to design and develop a more structured template to record lessons learned as the current free-flow text does not capture the full extent of what has been learned. An initial design has been provided that, once implemented, can be integrated within the company's existing PLM system. For future works, the system should be implemented to validate our approach. In addition a case study with real life data should be tested.

Throughout the industrial investigation of the Company, there is a requirement to make the maintenance process clearer to all the stakeholders through the product maintenance cycle. Besides, the knowledge of product maintenance should be integrated with other stages of the product lifecycle, such as NPD. Further research will be carried out to develop an e-Maintenance system to enhance the current PSS management.

\section{ACKNOWLEDGEMENTS}

The authors would like to thank all at the collaborating company for their support with this project and their input to the Industrial Investigation. This research is based on work funded by the European Program INTERREG IVA France-Channel-UK under the project entitled "Building an Expertise Network for an Efficient Innovation and Training System (BENEFITS)", under the INTERREG Programme France (Channel) - England, Ref 4177.

\section{REFERENCES}

[1] Ardichvili, A.; Maurer, M.; Li, W.; Wentling, T.; Stuedemann, R: Cultural influences on knowledge sharing through online communities of practice, Journal of Knowledge Management, 10(1), 2006, 94-107. http://dx.doi.org/10.1108/13673270610650139

[2] Argote, L.; Beckman, S.L.; Epple, D.: The persistence and transfer of learning in industrial settings. Journal of Management Science. 1990, 36 (2), 140-154. http://dx.doi.org/10.1287/mnsc.36.2.140

[3] Badria, A.; Niazib, A. N.; Hoseinic, S. M.: Long term preventive generation maintenance scheduling with network constraints, Energy Procedia, 2012, 14, 1889-1895.

http://10.1016/j.egypro.2011.12.887

[4] Bartlett, C.A.; Ghoshal, S.: Managing Across Borders: The Transnational Solution. 2nd. Boston, MA, USA: Harvard Business School Press, 1998.

[5] Beckman, T.: A Methodology For Knowledge Management. International Association of Science and Technology for Development AI and Soft Computing Conference. Banff, Canada: 27th July 1st August, 1997. 
[6] Bradfield, D.J. A Prototype Method and Tool to Facilitate Knowledge Sharing in the New Product Development Process, in School of Applied Sciences, Cranfield University: Cranfield, UK, 2012, 401.

[7] Cantamessa, M.; Montagna, F.; Neirotti, P: An empirical analysis of the PLM implementation effects in the aerospace industry, Computers in Industry 2012, 63, 243-251.

http://dx.doi.org/10.1016/j.compind.2012.01.004

[8] Chebel-Morello, B.; Medjaher, K.; Arab, A. H.; Bandou, F.;Bouchaib, S.; Zerhouni, N.: E-Maintenance for photovoltaic power generation system, Energy Procedia, 18, 2012, 640-643. http://dx.doi.org/10.1016/j.egypro.2012.05.077

[9] Chirumalla, K.: Towards a Methodology for Lessons Learned Practice in Complex Product Development. Proceedings of the 15th European conference on Knowledge Management: ECKM2014. Academic Conferences and Publishing International, 2014,3.

[10] Choi, S.Y.; Kang, Y.S.; Lee, H.: The effects of socio-technical enablers on knowledge sharing: an exploratory examination. Journal of Information Science. 2008,34. http://10.1177/0165551507087710

[11] Choudhary, A. K.; Oluikpe, P. I.; Harding, J. A.; Carrillo, P. M.: The needs and benefits of Text Mining applications on Post-Project Reviews. Comput Ind., 2009, 60, 728-740. http://dx.doi.org/10.1016/j.compind.2009.05.006

[12] Cooke-Davies, T.: The "real" success factors on projects. International Journal of Project Management, 2002, 20, 185-190. http://dx.doi.org/10.1016/S0263-7863(01)00067-9

[13] Dahal, K. P.; Chakpitak, N.: Generator maintenance scheduling in power systems using metaheuristic-based hybrid approaches, Electric Power Systems Research, 2007, 77(7),771-779. http://dx.doi.org/10.1016/j.epsr.2006.06.012

[14] Desouza, K.; Evaristo, R.: Global Knowledge Management Strategies. European Management Journal, 2003, 21(1), 62-67. http://dx.doi.org/10.1016/S0263-2373(02)00152-4

[15] Dutta, D.; Ameri, F. Product lifecycle management: closing the knowledge loops. Journal of Computer-Aided Design \& Applications, 2005, 2(5), 577-590.

http://dx.doi.org/10.1080/16864360.2005.10738322

[16] Evans, R.; Gao, J.; Owodunni, O.; Shah, S.; Mahdikhah, S.; Messaadia, M.; Baudry, D.: A Framework for Improving the Sharing of Manufacturing Knowledge through Micro-Blogging. Advances in Production Management Systems. Innovative and Knowledge-Based Production Management in a Global-Local World, Springer Berlin Heidelberg, 2014, 19-26. http://dx.doi.org/10.1007/978-3662-44739-0_3

[17] Danesi, F.; Gardan, N.; Gardan,Y.; Reimeringer, M.: P4LM: A methodology for product lifecycle management, Computers in Industry, 59, 2008, 304-317.

http://dx.doi.org/10.1016/j.compind.2007.06.013

[18] Goedkoop, M. J.,van Halen, J. G.,Riele, H. T. and Rommens, P. J. M.: Product service systems, ecological and economic basics, Hague, The Netherlands, 1999.

[19] Goffin, K.; Koners, U.: Tacit knowledge, lessons learnt, and new product development. Journal of Product Innovation Management 28(2), 2011, 300-318. http://dx.doi.org/10.1111/j.15405885.2010.00798.x

[20] Grieves, M: Product Lifecycle Management: Driving the Next Generation of Lean Thinking, McGraw-Hill Education, 2006

[21] Holubowicz, W.; Dymowski, W,; Springer, T.: Managing Lessons Learnt from daily missions: Methodology and tool. Gdansk, Poland: IEEE Computer Society. (2012)

[22] Jackson, I.; Humphrey, J.; Carrie, M.; Hodgson, M.; Binns, J.; Document \& Record Management Strategy, BAE Systems, 2008.

[23] Latify, M. A.; Seifi, H.; Rajabi M, H.: An integrated model for generation maintenance coordination in a restructured power system involving gas network constraints and uncertainties, International Journal Of Electrical Power \& Energy Systems, 46, 2013, 425-440. http://dx.doi.org/10.1016/j.ijepes.2012.10.017

[24] Liebowitz, J.: Knowledge management handbook. Florida, USA: CRC Press Inc, 1999.

[25] Liu, A.; Wuest, T.;Wei, W.; Lu, S.: Application of prospect theory on car sharing product service system, Procedia CIRP, 16, 2014, 350-355. http://dx.doi.org/10.1016/j.procir.2014.02.022 
[26] Mabert, V.A.; Soni, A.K.; Venkataramanan, M.A.: The impact of organization size on enterprise resource planning (ERP) implementations in the US manufacturing sector, OMEGA 31, 2003, 235246. http://dx.doi.org/10.1016/S0305-0483(03)00022-7

[27] Milton, N. Exploring the concept of organizational learning. KM Review, 2008, 11(4), 8-13.

[28] Milton, N. The Lessons Learned Handbook: Practical Knowledge-Based Approach to Learning from Experience, Oxford: Chandos Publishing Ltd.,2010.

http://dx.doi.org/10.1533/9781780631929

[29] Moon, S. K.; Simpson, T. W.; Shu, J.; Kumara, S. R. T.: Service representation for capturing and reusing design knowledge in product and service families using object-oriented concepts and an ontology, Journal Of Engineering Design, 20(4), 2009, 413 - 431.

http://dx.doi.org/10.1080/09544820903151723

[30] Muller, A.; Crespo Marquez, A.;Iung, B.: On the concept of e-maintenance: Review and current research, Reliability Engineering \& System Safety, 93(8), 2008, 1165-1187. http://dx.doi.org/10.1016/j.ress.2007.08.006

[31] Myers, M.B.; Cheung, M.: Sharing Global Supply Chain Knowledge, 2008. http://sloanreview.mit.edu/article/sharing-global-supply-chain-knowledge.

[32] Nonaka, I.: The Knowledge-Creating Company. Harvard Business Review. Vol. 69: pp. 96-104, 1991.

[33] Nonaka, I.; Takeuchi, H.:The Knowledge-Creating Company. Oxford University Press, NewYork, 1995.

[34] Nunamaker, J.F.; Romano, N.C.; Briggs, R.O.: A Framework for Collaboration and Knowledge Management. 34th Hawaii International Conference on System Sciences. Maui, Hawaii, United States: January, 2001,1-12.

[35] Pillai, K.G.; Min, S.: A Firm's Capability to Calibrate Supply Chain Knowledge - Antecendents and Consequences. International Journal of Industrial Marketing Management, 39(8), 2010, 1365 1375. http://dx.doi.org/10.1016/j.indmarman.2010.04.002

[36] Pol, G., Merlo, C.; Legardeur, J.: Implementation of collaborative design processes into PLM systems. International Journal of Product Lifecycle Management, 2008, 3(4), 279-294. http://dx.doi.org/10.1504/IJPLM.2008.027006

[37] Porter, M.E. and Millar, V.E. "How Information Gives You Competitive Advantage". Harvard Business Review, 1985, 63(4), 149-160.

[38] Preiss, K.J. "A two-stage process for eliciting and prioritising critical knowledge". Journal of Knowledge Management, 2000, 4(4), 328-336. http://dx.doi.org/10.1108/13673270010379885

[39] Rebolledo, C. and Nollet, J. "Learning from suppliers in the aerospace industry". International Journal on Production Economics, 2011, 129(2), 328-337.

http://dx.doi.org/10.1016/j.ijpe.2010.11.008

[40] Richter, MM and Weber RO. Case-Based Reasoning: A Textbook. Springer-Verlag Berlin Heidelberg, 2013. http://dx.doi.org/10.1007/978-3-642-40167-1

[41] Riege, A.: Three-dozen knowledge-sharing barriers managers must consider. Journal of Knowledge Management, 2005, 9(3), 18-35. http://dx.doi.org/10.1108/13673270510602746

[42] Ross, D.F. 1998. Competing through supply chain management. New York, USA: Chapman \& Hall. http://dx.doi.org/10.1007/978-1-4757-4816-1

[43] Schindler, M.; Eppler, M.J.: Harvesting project knowledge: A review of project learning methods and success factors. International Journal of Project Management, 2003, 21, 219-228. http://dx.doi.org/10.1016/S0263-7863(02)00096-0

[44] Secchi, P.; Ciaschi, R.; Spence, D.: A concept for an ESA lessons learned system. In: Proceedings of the 5th Annual International Symposium of the National Council on System Engineering (INCOSE), St. Louis, Missouri, 1999.

[45] Shin, M.; Holden, T.; Schmidt, R.A.: From knowledge theory to management practice: Towards an integrated approach. Journal of Information Processing and Management, 2001, 37(2), 335-355. http://dx.doi.org/10.1016/S0306-4573(00)00031-5

[46] Srinivasan, V: An integration framework for product lifecycle management, Computer (V.A. Mabert, 2003)-Aided Design, 2011, 43(5), 464-478. http://dx.doi.org/10.1016/j.cad.2008.12.001

[47] Tian, Z.; Jin, T.; Wu, B.; Ding, F.: Condition based maintenance optimization for wind power generation systems under continuous monitoring, Renewable Energy, 2011, 36(5),1502-1509. http://dx.doi.org/10.1016/j.renene.2010.10.028 
[48] Tsoukas, H. and Vladimirou, E. "What is organizational knowledge?". Journal of Management Studies, 2001, 38(7), 973-993. http://dx.doi.org/10.1111/1467-6486.00268

[49] Zhu, Q.; Jiang, P.: An outsourcing e-maintenance system for improving maintenance of an industrial product service system, Applied Mechanics and Materials, 2009, 1077-1081.

[50] Zhu, Q. Q.; Jiang, P. Y.; Huang, G. Q.; Qu, T.: Implementing an industrial product-service system for CNC machine tool, The International Journal of Advanced Manufacturing Technology, 2011, 52(9-12), 1133-1147. http://dx.doi.org/10.1007/s00170-010-2761-9

[51] Wan, S.; Gao, J.; Li, D.B.; Evans, R.: Knowledge management for maintenance, repair and service of manufacturing system. 12th International Conference on Manufacturing Research (ICMR2014). Southampton, England, the United Kingdom: September, 2014, 65-70. 